\title{
Prognostic Impact of Proximal Versus Distal Localization in Extremity Long Bone Osteosarcomas
}

\author{
KJETIL BERNER $^{1}$ and $Ø$ YVIND S. BRULAND $^{1,2}$ \\ ${ }^{1}$ Department of Oncology, Oslo University Hospital, The Norwegian Radium Hospital, Oslo, Norway; \\ ${ }^{2}$ Institute for Clinical Medicine, Faculty of Medicine, University of Oslo, Oslo, Norway
}

\begin{abstract}
Background/Aim: This study aimed to identify the prognostic factors and outcomes of osteosarcoma (OS) located in proximal versus distal extremity long bones. Patients and Methods: A nationwide cohort comprising all Norwegian highgrade OSs in extremity long bones between 1982 and 2009 was investigated. Results: The univariate analysis results identified no significant differences in survival between patients with $O S$ in proximal long bones (101 cases) as a group in comparison to patients with $O S$ in the distal part of these bones (120 cases). However, proximal femur and primary metastasis were both independent adverse prognostic factors for sarcomaspecific survival in multivariate analyses, while elevated $L D H$ and secondary OS were inferior prognostic factors for eventfree survival. Adequate surgery and chemotherapy had a positive impact on survival. Conclusion: OS of the proximal femur had an unfavorable outcome in comparison to OS in other anatomical locations in extremity long bones.
\end{abstract}

It is well established that axial tumor locations of osteosarcoma (OS) result in a worse outcome than primary disease located in the appendicular skeleton (1-5). Proximal osteosarcoma in extremity long bones (POS) has also been associated with poor outcome $(4,6-8)$, although the prognostic impact of the anatomical location of such tumors is still debated $(6,8,9)$. Hence, this study aimed to evaluate the clinical features and outcomes of POS versus distal osteosarcoma in extremity long bone (DOS) in a nationwide Norwegian OS population. To the best of our knowledge, clinical epidemiology and treatment outcomes of POS versus

This article is freely accessible online.

Correspondence to: Kjetil Berner, MD, Ph.D., Department of Oncology, Oslo University Hospital, The Norwegian Radium Hospital, P.O. Box 4953, Nydalen, N-0424 Oslo, Norway. Tel: +47 22934000, Fax: +47 22525559, e-mail: kjetil.berner@ous-hf.no

Key Words: Prognostic factors, overall survival, population-based, osteosarcoma, pathological fracture.
DOS have not been previously reported in such a complete and population-based cohort.

\section{Materials and Methods}

Patient cohort. We analyzed 221 cases of extremity long bone OS identified among the 335 histologically-verified high-grade OS patients diagnosed in Norway between 1982 and 2009. The gross cohort included all subgroups of OS, even secondary OS (10), according to the current World Health Organization criteria (11). The following variables were relevant to this study: retrospectively validated cases based on multiple and partly overlapping data from registry sources, including all cases reported to the Norwegian Cancer Registry (NCR), supplemented with clinical records from all Norwegian hospitals involved in sarcoma management (10).

The starting year of 1982 was in line with the introduction of modern, multi-agent chemotherapy in Norway (12). The ending year of 2009 was the last available year of registration of primary diagnosis according to the NCR when the current project began. All follow-up data were updated to the second half of 2013.

Demographic and tumor-related variables. The demographic and tumor-related variables are presented in Table I. Tumor size was defined as the maximum length of the tumor in centimeters $(\mathrm{cm})$; duration of symptoms was defined as the interval in months between the first symptom and the time of biopsy. Tumor size above $11 \mathrm{~cm}$ and duration of symptoms longer than three months were defined as elevated values in the analyses, and metastasis that was evident within six weeks of primary diagnosis was defined as primary metastatic disease. Serum alkaline phosphatase (ALP) and serum lactate dehydrogenase $(\mathrm{LDH})$ were measured in international units at the time of diagnosis. Data on the date and cause of death were primarily retrieved from the Cause of Death Registry (10).

Treatment variables. Adequate treatment was defined as having undergone surgery for all detectable disease in addition to chemotherapy. Adequate surgery implied surgical removal of the primary tumor with wide or marginal margins as described by Enneking et al. (13), while adequate chemotherapy was defined as having received at least six courses of chemotherapy. The latter definition with its justification, is more thoroughly presented in a previous study (5). Patients with metastatic disease at the time of diagnosis were in need for a surgical remission for both primary tumor and metastases in order to be classified as having undergone adequate surgical treatment. 
Table I. Characteristics of patients with proximal osteosarcoma in long bones (POS) and distal osteosarcoma localization (DOS).

\begin{tabular}{|c|c|c|c|c|}
\hline & All patients $(\%)$ & $\operatorname{POS}(\%)^{1}$ & $\operatorname{DOS}(\%)^{1}$ & $p$-Value ${ }^{2}$ \\
\hline All patients & $221(100)$ & $101(46)$ & $120(54)$ & \\
\hline Gender & & & & 0.371 \\
\hline Male & $134(61)$ & $58(43 / 57)$ & $76(57 / 63)$ & \\
\hline Female & 87 (39) & $43(49 / 43)$ & $44(51 / 37)$ & \\
\hline Anatomic site & & & & $<0.001$ \\
\hline Femur & $125(57)$ & $18(14 / 18)$ & $107(86 / 89)$ & \\
\hline Tibia & $58(26)$ & $50(86 / 50)$ & $8(14 / 7)$ & \\
\hline Fibula & $10(4)$ & $10(100 / 10)$ & $0(0 / 0)$ & \\
\hline Humerus & $24(11)$ & $22(92 / 22)$ & $2(8 / 2)$ & \\
\hline Radius/ulna & $4(2)$ & $1(25 / 1)$ & $3(75 / 3)$ & \\
\hline Age & & & & 0.566 \\
\hline$\leq 20$ years & $138(62)$ & $66(48 / 65)$ & $72(52 / 60)$ & \\
\hline $20-40$ years & $44(20)$ & $17(39 / 17)$ & $27(61 / 23)$ & \\
\hline$>40$ years & $39(18)$ & $18(46 / 18)$ & $21(54 / 17)$ & \\
\hline Tumor size 3,4 & & & & 0.476 \\
\hline$\leq 11 \mathrm{~cm}$ & $112(57)$ & $49(44 / 54)$ & $63(56 / 59)$ & \\
\hline$>11 \mathrm{~cm}$ & $86(43)$ & $42(49 / 46)$ & $44(51 / 41)$ & \\
\hline Duration of symptoms $s^{3,5}$ & & & & 0.319 \\
\hline$\leq 3$ months & $83(52)$ & $35(42 / 47)$ & $48(58 / 55)$ & \\
\hline$>3$ months & $78(48)$ & $39(50 / 53)$ & $39(50 / 45)$ & \\
\hline Pathological fracture & & & & 0.742 \\
\hline No & $184(83)$ & $85(46 / 84)$ & $99(54 / 83)$ & \\
\hline Yes & $37(17)$ & $16(43 / 16)$ & $21(57 / 17)$ & \\
\hline Primary metastasis ${ }^{3}$ & & & & 0.388 \\
\hline No & $169(77)$ & $75(44 / 74)$ & $94(56 / 79)$ & \\
\hline Yes & $51(23)$ & $26(51 / 26)$ & $25(49 / 21)$ & \\
\hline Secondary osteosarcoma & & & & 0.635 \\
\hline No & $213(96)$ & $98(46 / 97)$ & $115(54 / 96)$ & \\
\hline Yes & $8(4)$ & $3(38 / 3)$ & $5(62 / 4)$ & \\
\hline $\mathrm{LDH}^{3}$ & & & & 0.794 \\
\hline Normal & $82(54)$ & $41(50 / 55)$ & $41(50 / 53)$ & \\
\hline Elevated & $71(46)$ & $34(42 / 45)$ & $37(52 / 47)$ & \\
\hline $\mathrm{ALP}^{3}$ & & & & 0.162 \\
\hline Normal & $90(56)$ & $48(53 / 62)$ & $42(47 / 51)$ & \\
\hline Elevated & $71(44)$ & $30(42 / 38)$ & $41(58 / 49)$ & \\
\hline Histologic type ${ }^{3}$ & & & & 0.216 \\
\hline Osteoblastic & $106(49)$ & $48(45 / 49)$ & $58(55 / 49)$ & \\
\hline Chondroblastic & $25(12)$ & $8(32 / 8)$ & $17(68 / 14)$ & \\
\hline Fibroblastic & $20(9)$ & $8(40 / 8)$ & $12(60 / 10)$ & \\
\hline Mixed & $57(26)$ & $28(49 / 28)$ & $29(51 / 25)$ & \\
\hline Other & $9(4)$ & $7(78 / 7)$ & $2(22 / 2)$ & \\
\hline Years of diagnosis & & & & 0.327 \\
\hline $1982-1989$ & $68(31)$ & $30(44 / 30)$ & $38(56 / 32)$ & \\
\hline 1990-1999 & $70(32)$ & $28(40 / 28)$ & $42(60 / 35)$ & \\
\hline 2000-2009 & $83(38)$ & $43(52 / 42)$ & $40(48 / 33)$ & \\
\hline Surgical procedure ${ }^{3}$ & & & & 0.064 \\
\hline Amputation & $74(36)$ & $26(35 / 29)$ & $48(65 / 41)$ & \\
\hline Other & $132(64)$ & $64(48 / 71)$ & $68(52 / 59)$ & \\
\hline Adequate surgery & & & & 0.193 \\
\hline Yes & $183(83)$ & $80(44 / 79)$ & $103(56 / 86)$ & \\
\hline No surgery & $13(6)$ & $9(69 / 9)^{6}$ & $4(31 / 3)$ & \\
\hline Inadequate surgery & $25(11)$ & $12(48 / 12)$ & $13(52 / 11)$ & \\
\hline Adequate chemotherapy & & & & 0.324 \\
\hline Yes & $182(82)$ & $86(47 / 85)$ & $96(53 / 80)$ & \\
\hline No & $39(18)$ & $15(38 / 15)$ & $24(62 / 20)$ & \\
\hline Adequate treatment & & & & 0.846 \\
\hline Yes & $163(74)$ & $76(47 / 75)$ & $87(53 / 73)$ & \\
\hline No & $58(26)$ & $25(43 / 25)$ & $33(57 / 27)$ & \\
\hline
\end{tabular}

${ }^{1}$ Both row percentage (nominator) and column percentage (denominator). ${ }^{2} \chi^{2} \cdot{ }^{3}$ Missing values equal the difference between the summarized numbers in the second column and the total patients in the study. ${ }^{4}$ Median tumor size was $11 \mathrm{~cm}$. ${ }^{5}$ Median duration of symptoms before biopsy was 3 months. ${ }^{6}$ Eight cases located to proximal femur and one in proximal humerus, mainly due to primary metastatic disease. 


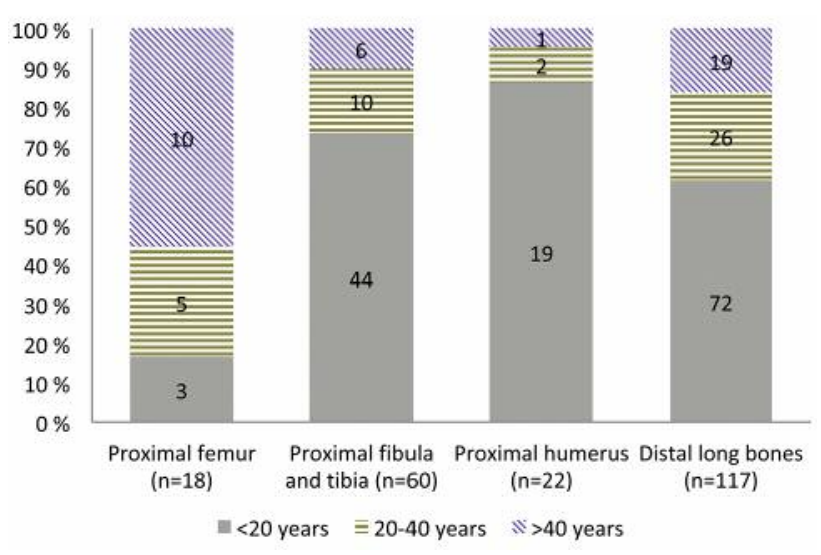

Figure 1. Distribution of age and primary site in extremity long bone osteosarcomas at diagnoses (femur, humerus, tibia and fibula).

Statistical analyses. Chi square analyses $\left(\chi^{2}\right)$ were used to compare unrelated samples, when appropriate. Survival analyses using Kaplan-Meier estimates, the log-rank test, and Cox regression were used to analyze overall survival, sarcoma specific survival (SSS), and event-free survival (EFS), using SPSS version 21 (SPSS Inc., Chicago, IL, USA) and Stata version 13.1 (Stata corporation, College Station, TX, USA) software. The endpoint for all survivors in the entire cohort was set at July 2013. Overall survival was calculated from the date of diagnosis until the date of death from any cause, while sarcoma-specific death or treatment-related death was the endpoint of SSS. EFS was calculated from the date of diagnosis until the date of the first metastasis, local recurrence, sarcoma-specific death, or treatment-related death, whichever occurred first. Patients with primary metastatic OS were not included in the EFS analyses. The statistically significant prognostic variables in the univariate analysis were included in the multivariate backward Cox-regression analyses. The Cox proportion hazard assumption was evaluated using Kaplan-Meier plots. We did not account for multiple imputations of missing values in this report, since their effects were considered to be modest (5).

Ethical approval. The Regional Ethical Committee was informed about this project, although the study did not require formal ethical approval since the data registration was in line with the legitimate mandate of the NCR.

\section{Results}

Anatomical localization. This study included 101 (46\%) POS patients and 120 (54\%) DOS patients (Table I). Proximal anatomical locations were more common in the fibula $(100 \%)$, humerus $(92 \%)$, tibia $(86 \%)$, and radius/ulna $(25 \%)$ than in the femur, where only $14 \%$ of the tumors were proximally located $(p<0.001)$. We observed a substantially higher percentage of OS located in the proximal femur among elderly patients in contrast to other anatomical locations in long bones (Figure 1).

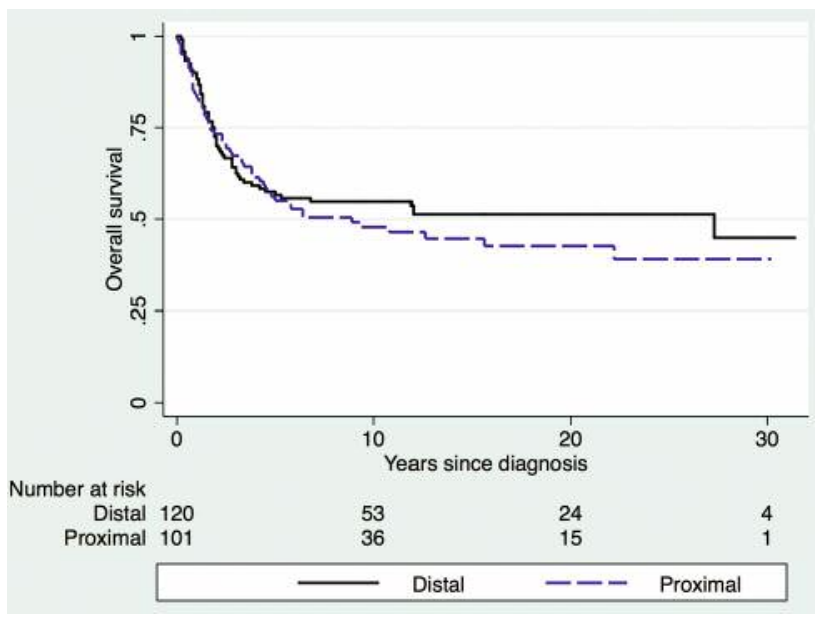

Figure 2. Overall survival of patients with osteosarcoma in proximal and distal long bones $(p=0.418)$.

Clinicopathological data. As seen in Table I, there were no significant differences between the POS and the DOS groups regarding gender, age, tumor size, duration of symptoms, pathological fracture, secondary OS, LDH, ALP, and histologic type. Approximately 23\% (51 patients) of all patients had metastatic disease at the time of OS diagnosis, nearly equally distributed among groups of POS and DOS, respectively. In contrast, as many as $44 \%$ (8 cases) of the patients with OS in the proximal femur had primary metastatic disease.

Treatment. Amputations were more often performed in DOS patients $(65 \%)$ in comparison to the POS patients (35\%) (Table I). We have previously demonstrated a significant increase in limb salvage procedures since the 1980s for high-grade OS in Norway during this time period, although amputation still remains a valid and justified procedure in selected OS cases (5). In the current study, we documented a higher percentage of adequate surgery among patients with DOS (86\%) as compared to those having POS (79\%) (Table I). Interestingly, only 39\% (7 patients) of the cases with a primary tumor in the proximal femur received adequate surgery in comparison to all (10 patients) of the cases with a primary tumor in the fibula, $88 \%$ (44 patients) of the cases with a primary tumor in the tibia and $82 \%$ (18 patients) with a primary tumor in the humerus. This discrepancy was due to the fact that eight patients in the previously mentioned POS subgroup did not undergo surgery, mainly due to disseminated disease at the time of diagnosis and/or higher age (Figure 1). We observed no significant differences in receiving adequate treatment among the patients in the POS versus the DOS on a group basis $(p=0.846)$, as presented in Table I. 
Table II. Univariate Kaplan-Meier and Cox-regression analyses of five-year sarcoma specific survival and event-free survival based on different characteristics of long bone osteosarcoma.

\begin{tabular}{|c|c|c|c|c|c|c|c|c|}
\hline & \multicolumn{4}{|c|}{ Sarcoma specific survival } & \multicolumn{4}{|c|}{ Event-free survival } \\
\hline & $\begin{array}{c}\text { Patients } \\
(\%)\end{array}$ & $\begin{array}{c}5 \text { years in } \% \\
\left(95 \% \mathrm{CI}^{1} \text { in } \%\right)\end{array}$ & $\begin{array}{c}\mathrm{RR}^{2} \\
\left(95 \% \mathrm{CI}^{1}\right)\end{array}$ & $p$-Value ${ }^{3}$ & $\begin{array}{l}\text { Patients } \\
(\%)\end{array}$ & $\begin{array}{c}5 \text { years in } \% \\
\left(95 \% \mathrm{CI}^{1} \text { in } \%\right)\end{array}$ & $\begin{array}{c}\mathrm{RR}^{2} \\
\left(95 \% \mathrm{CI}^{1}\right)\end{array}$ & $p$-Value ${ }^{3}$ \\
\hline Gender & & & & 0.455 & & & & 0.031 \\
\hline Male & $134(61)$ & $56(48-64)$ & 1 & & $109(64)$ & $51(42-60)$ & 1 & \\
\hline Female & 87 (39) & $59(47-68)$ & $0.9(0.6-1.3)$ & & $60(36)$ & $68(55-78)$ & $0.6(0.4-1.0)$ & \\
\hline Anatomic site & & & & 0.001 & & & & 0.072 \\
\hline Distal femur & $107(48)$ & $58(47-66)$ & 1 & & $82(49)$ & $61(48-70)$ & 1 & \\
\hline Proximal femur & $18(8)$ & $22(7-43)$ & $3.7(2.1-6.8)$ & $<0.001$ & $9(5)$ & $33(8-62)$ & $2.5(1.1-5.7)$ & 0.026 \\
\hline Proximal tibia & $50(23)$ & $70(55-81)$ & $0.5(0.5-1.4)$ & 0.468 & $42(25)$ & $62(45-74)$ & $0.9(0.5-1.5)$ & 0.594 \\
\hline Distal tibia & $8(4)$ & $63(23-86)$ & $1.1(0.4-3.2)$ & 0.803 & $7(4)$ & $14(1-46)$ & $2.5(1.1-6.1)$ & 0.035 \\
\hline Proximal fibula & $10(5)$ & $48(16-75)$ & $1.0(0.4-2.5)$ & 0.994 & $7(4)$ & $69(21-92)$ & $0.8(0.2-2.6)$ & 0.707 \\
\hline Proximal humerus & $22(10)$ & $55(32-72)$ & $1.3(0.7-2.4)$ & 0.417 & $16(9)$ & $56(30-76)$ & $1.2(0.6-2.6)$ & 0.567 \\
\hline Other & $6(3)$ & $67(20-90)$ & $1.1(0.3-3.4)$ & 0.927 & $6(4)$ & $67(20-90)$ & $1.1(0.3-3.6)$ & 0.875 \\
\hline Age & & & & 0.003 & & & & $<0.001$ \\
\hline$\leq 20$ years & $138(62)$ & $63(54-71)$ & 1 & & $111(66)$ & $74(65-81)$ & 1 & \\
\hline $20-40$ years & $44(20)$ & $56(40-69)$ & $1.2(0.8-2.0)$ & 0.394 & $30(18)$ & $73(53-85)$ & $1.2(0.6-2.2)$ & 0.585 \\
\hline$>40$ years & $39(18)$ & $39(24-53)$ & $2.3(1.4-3.6)$ & 0.001 & $28(17)$ & $36(19-53)$ & $3.3(1.9-5.6)$ & $<0.001$ \\
\hline Tumor size & & & & 0.011 & & & & 0.090 \\
\hline$\leq 11 \mathrm{~cm}$ & $86(43)$ & $66(57-75)$ & 1 & & $60(39)$ & $63(53-72)$ & 1 & \\
\hline$>11 \mathrm{~cm}$ & $112(57)$ & $45(37-52)$ & $1.7(1.1-2.6)$ & & $95(61)$ & $48(35-60)$ & $1.5(0.9-2.3)$ & \\
\hline Pathological fracture & & & & 0.048 & & & & 0.041 \\
\hline No & $184(83)$ & $60(53-67)$ & 1 & & $143(85)$ & $59(51-67)$ & 1 & \\
\hline Yes & 37 (17) & $43(27-58)$ & $1.6(1-2.6)$ & & $26(15)$ & $46(27-64)$ & $1.8(1.0-3.0)$ & \\
\hline Primary metastasis & & & & $<0.001$ & & & & \\
\hline No & $169(77)$ & $69(61-76)$ & 1 & & & & & \\
\hline Yes & $51(23)$ & $17(8-29)$ & $4.2(2.8-6.3)$ & & & & & \\
\hline Secondary osteosarcoma & & & & 0.116 & & & & $<0.048$ \\
\hline No & $213(96)$ & $58(51-64)$ & 1 & & $163(96)$ & $58(50-65)$ & 1 & \\
\hline Yes & $8(4)$ & $38(9-67)$ & $2.0(0.8-5.0)$ & & $6(4)$ & $33(5-68)$ & $2.4(1.0-6.0)$ & \\
\hline LDH & & & & 0.005 & & & & 0.037 \\
\hline Normal & $82(54)$ & $69(58-78)$ & 1 & & $68(58)$ & $63(52-74)$ & 1 & \\
\hline Increased & $71(46)$ & $45(33-56)$ & $1.9(1.2-3.1)$ & & $49(42)$ & $49(35-62)$ & $1.7(1.0-2.9)$ & \\
\hline ALP & & & & $<0.001$ & & & & 0.005 \\
\hline Normal & $90(56)$ & $71(60-79)$ & 1 & & $80(65)$ & $66(55-75)$ & 1 & \\
\hline Increased & $71(44)$ & $39(28-50)$ & $2.3(1.4-3.5)$ & & $43(35)$ & $42(27-56)$ & $2.1(1.2-3.4)$ & \\
\hline Histological subtype & & & & $<0.081$ & & & & 0.251 \\
\hline Osteoblastic & $106(49)$ & $53(43-62)$ & 1 & & $80(48)$ & $54(42-64)$ & 1 & \\
\hline Chondroblastic & $25(11)$ & $76(54-88)$ & $0.6(0.3-1.2)$ & 0.117 & $21(13)$ & $62(38-79)$ & $0.8(0.4-1.6)$ & 0.765 \\
\hline Fibroblastic & $20(9)$ & $75(50-89)$ & $0.5(0.2-1.1)$ & 0.486 & $15(9)$ & $60(32-80)$ & $0.9(0.4-1.9)$ & 0.869 \\
\hline Mixed & $58(27)$ & $57(43-69)$ & $0.6(0.7-1-3)$ & 0.882 & $47(28)$ & $60(44-72)$ & $1.0(0.6-1.6)$ & 0.918 \\
\hline Other & $9(4)$ & $11(1-39)$ & $3.8(1.9-7.7)$ & $<0.001$ & $3(2)$ & $33(1-77)$ & $3.2(1.0-10.3)$ & 0.056 \\
\hline Years of diagnosis & & & & 0.039 & & & & 0.057 \\
\hline $1982-89$ & $68(31)$ & $47(33-57)$ & $1.8(1.1-2.9)$ & 0.015 & $51(30)$ & $41(28-54)$ & $1.9(1.1-3.2)$ & 0.022 \\
\hline 1990-99 & $70(32)$ & $59(46-69)$ & $1.2(0.8-2.0)$ & 0.396 & $54(32)$ & $59(45-71)$ & $1.3(0.7-2.2)$ & 0.416 \\
\hline $2000-09$ & $83(37)$ & $65(54-74)$ & 1 & & $64(38)$ & $69(56-79)$ & 1 & \\
\hline Adequate treatment & & & & $<0.001$ & & & & $<0.001$ \\
\hline Yes & $163(74)$ & $72(65-78)$ & 1 & & $139(82)$ & $67(58-74)$ & 1 & \\
\hline No & $58(26)$ & $15(7-25)$ & $6.7(4.5-10)$ & & $30(18)$ & $11(3-25)$ & $9.2(5.6-15.2)$ & \\
\hline
\end{tabular}

${ }^{1}$ Confidence interval, ${ }^{2}$ relative risk and ${ }^{3} \log$ rank.

Metastatic relapse or local recurrence during follow-up. Among the patients without primary metastatic disease, 30 of the POS patients $(42 \%)$ and 41 of the DOS patients (44\%) developed metastases from OS during follow-up $(p=0.636)$.
Approximately $90 \%$ of the patients in both metastatic subgroups developed recurrence in the lungs (first metastatic relapse). Among the patients with no primary metastatic disease, four patients experienced local relapse during 
Table III. Multivariate Cox-regression analyses of prognostic factors and treatment-related variables for sarcoma specific survival and eventfree survival.

\begin{tabular}{|c|c|c|c|c|}
\hline \multirow[b]{2}{*}{ Variables $^{1}$} & \multicolumn{2}{|c|}{ Sarcoma specific survival } & \multicolumn{2}{|c|}{ Event-free surviva } \\
\hline & $\begin{array}{c}\mathrm{RR}^{2} \\
\left(95 \% \mathrm{CI}^{3}\right)\end{array}$ & $p$-Value & $\begin{array}{c}\mathrm{RR}^{2} \\
\left(95 \% \mathrm{CI}^{3}\right)\end{array}$ & $p$-Value \\
\hline Gender & & & $1.7(0.9-3.3)$ & 0.087 \\
\hline \multicolumn{5}{|l|}{ Anatomic site } \\
\hline Proximal femur & $5.5(2.3-13.1)$ & $<0.001$ & & \\
\hline Proximal tibia & $1.1(0.6-2.1)$ & 0.776 & & \\
\hline Distal tibia & $0.7(0.2-3.1)$ & 0.623 & & \\
\hline Proximal fibula & $1.3(0.4-3.9)$ & 0.618 & & \\
\hline Proximal humerus & $1.4(0.6-3.1)$ & 0.427 & & \\
\hline Other & $2.3(0.5-10.0)$ & 0.276 & & \\
\hline Primary metastasis & $3.9(2.3-6.6)$ & $<0.001$ & & \\
\hline Secondary osteosarcoma & & & $11.1(2.4-52.6)$ & 5) 0.002 \\
\hline Elevated LDH & $1.6(1.0-2.7)$ & 0.068 & $1.8(1.0-3.0)$ & 0.034 \\
\hline \multicolumn{5}{|l|}{ Years at diagnosis } \\
\hline $1982-1989$ & $2.1(1.1-3.7)$ & 0.015 & & \\
\hline 1990-1999 & $1.7(0.9-3.3)$ & 0.112 & & \\
\hline 2000-2009 & 1 & & & \\
\hline \multicolumn{5}{|l|}{ Adequate treatment } \\
\hline Yes & 1 & & 1 & \\
\hline No & $4.3(2.3-7.7)$ & $<0.001$ & $4.4(2.4-7.9)$ & $<0.001$ \\
\hline
\end{tabular}

${ }^{1}$ References values in line with Table II. ${ }^{2}$ Relative risk and ${ }^{3}$ confidence interval.

follow-up in both the POS (5\%) and the DOS (4\%) subgroups, respectively $(p=0.743)$. The median time to the first metastatic event, or local recurrence, was 2.2 years (range $=0.2-11.6$ years) and 2.3 years (range $=0.3-7.6$ years) from diagnosis, respectively.

Survival analysis. Univariate survival analyses revealed no difference in survival between all POS and DOS patients (Figure 2; SSS, $p=0.430$; EFS, $p=0.808$ ). Nevertheless, a significant discrepancy was observed in both overall survival and SSS within the respective groups of patients with OS in extremity long bones, with the poorest prognosis for patients with a primary tumor located in the proximal femur (Table II and Figure 3A). The poor prognosis of the latter subgroup regarding overall survival and SSS was independent of primary metastatic disease (Figure 3B, SSS, $p=0.037$ ) or not (Figure $3 \mathrm{C}$, SSS, $p=0.022$; EFS, $p=0.086$ ). We did not identify any corresponding difference in survival among the groups; neither in patients below 20 years at time of diagnosis (Figure 3D; SSS, $p=0.702$; EFS, $p=0.422$ ) nor among patients that were adequately treated (Figure 3E; SSS, $p=0.539$; EFS, $p=0.227$ ).

Table II presents the results of the univariate analyses as fiveyear SSS and EFS based on different OS characteristics, including time periods and adequate versus inadequate treatment. The non-significant impact of the duration of symptoms observed $(p=0.913 / 0.947)$ is not included in Table II.

Prognostic factors. The results of the final step in the multivariate cox analyses are presented in Table III. OS in proximal femur and primary metastatic disease were both adverse prognostic factors for SSS while elevated LDH and secondary OS were inferior prognostic factors for EFS. As expected, patients that received adequate treatment had significantly better SSS and EFS than patients that received inadequate treatment. No improvement in SSS was identified for all patients since the 1990s.

\section{Discussion}

No previous nationwide studies have analyzed the prognostic relevance of POS versus DOS. The current cohort of extremity long bone OS was extracted from an unselected cohort that comprised all subgroups and histopathological entities of OS, including secondary OS, between 1982 and 2009 in Norway.

We observed no difference in survival between all patients dichotomized between POS and DOS primary disease in extremity long bones (Figure 2). This finding is in accordance with the results reported in a previous study from the United Kingdom (9). However, several prior studies found poorer outcomes for POS than DOS $(4,6,14,15)$. Nevertheless, we documented inferior survival among all patients with OS in the proximal femur in comparison to OS in other anatomical locations in extremity long bones (Figure 3a, Table II, Table III). Previous reports have also documented the proximal femur as an unfavorable anatomical site for OS $(4,6,8,16)$.

The dismal outcome of proximal tumor sites documented in previous studies has been linked to several hypotheses, such as variations in chemosensitivity (8) or differences in other biological factors, such as regional blood flow (6). There is, however, no sound evidence for these hypotheses (6). The prognostic effect of the anatomical location of OS within a long bone can be influenced by methodological inequalities between various studies. For example, the proximal tibia and fibula were considered to be a distal site of origin in some prior studies $(4,8)$, while one study defined the correct anatomical location (6). Furthermore, in comparison to population-based studies, the results of prior studies may also depend on the clinical and demographic characteristics of the OS population being analyzed, which may be biased, for example, depending on the referral patterns, pediatric and adolescents versus including also adult OS patients and/or reporting systems of the various hospitals involved.

We believe that the strength of the present study is the reliability of the database, which is validated by multiple and partly overlapping data and registry sources. As expected in 

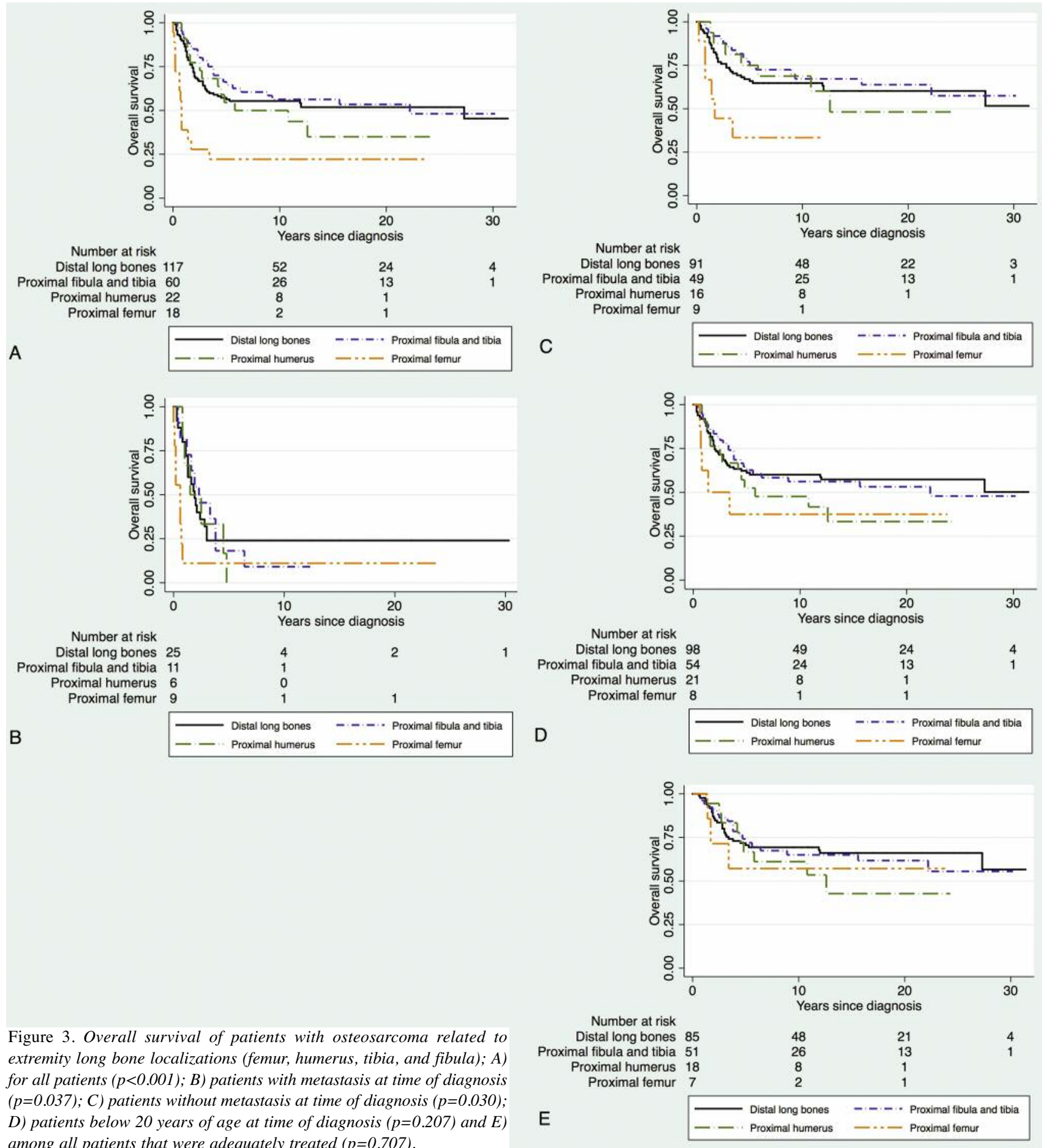

Figure 3. Overall survival of patients with osteosarcoma related to extremity long bone localizations (femur, humerus, tibia, and fibula); A) for all patients $(p<0.001) ; B)$ patients with metastasis at time of diagnosis ( $p=0.037)$; $C$ ) patients without metastasis at time of diagnosis ( $p=0.030$ ); $D)$ patients below 20 years of age at time of diagnosis $(p=0.207)$ and $E)$ among all patients that were adequately treated $(p=0.707)$.

nationwide studies, we were unable to obtain complete clinical information for all the patients in the present study. Moreover, unlike prospective clinical trials or experiences from institutional series, in our approach, it was not possible to obtain the same degree of detail regarding certain clinical variables. As an example, we cannot rule out that excluding histologic response to preoperative chemotherapy $(4,17,18)$ may have affected the prognostic factors presented in Table III. The latter would have necessitated a complete and uniform histological reexamination of all cases in the present 
cohort. Consequently, a significant disadvantage of such an approach is the lack of available histological specimens for reexamination. Hence, we have chosen not to include histological response to chemotherapy in this paper since we believe the potential disadvantage will exceed the potential gain of such an approach.

Furthermore, we were not able to include patients diagnosed with OS later than 2009 in the present cohort. However, we believe this limitation has minor impact on the results of the present study since conventional chemotherapy in conjunction with surgery has reached a plateau phase since the end of the 1980s $(2,19)$, also confirmed in this publication.

\section{Conclusion}

In this first study to investigate the prognostic importance of the anatomical location of OS within long bones of the appendicular skeleton in a nationwide setting. OS of the proximal femur had a more unfavorable outcome than OS in other locations in extremity long bones.

\section{Conflicts of Interest}

The Authors report no conflicts of interest. The Authors alone are responsible for the content and writing of the paper.

\section{Authors' Contributions}

Both Authors have contributed equally to concept and design of the study, data interpretation and writing the manuscript. First Author was responsible for establishing the gross database, merging clinical data from overlapping and multiple register sources and all data, as well as statistical analyses. Both Authors have in selected cases scrutinized available clinical records to ensure correct clinical information and approved the final version of the manuscript.

\section{Acknowledgements}

The Authors thank all colleagues within the multidisciplinary sarcoma teams at our and collaborating Institutions during 1982 and 2009.

\section{References}

1 Jawad MU, Cheung MC, Clarke J, Koniaris LG and Scully SP: Osteosarcoma: Improvement in survival limited to high-grade patients only. J Cancer Res Clin Oncol 137(4): 597-607, 2011. PMID: 20514491. DOI: 10.1007/s00432-010-0923-7

2 Whelan J, McTiernan A, Cooper N, Wong YK, Francis M, Vernon S and Strauss SJ: Incidence and survival of malignant bone sarcomas in england 1979-2007. Int J Cancer 131(4): E508-517, 2012. PMID: 21913189. DOI: 10.1002/ijc.26426

3 Saeter G, Hoie J, Stenwig AE, Johansson AK, Hannisdal E and Solheim OP: Systemic relapse of patients with osteogenic sarcoma. Prognostic factors for long term survival. Cancer 75(5): 1084-1093, 1995. PMID: 7850705.
4 Bielack SS, Kempf-Bielack B, Delling G, Exner GU, Flege S, Helmke K, Kotz R, Salzer-Kuntschik M, Werner M, Winkelmann W, Zoubek A, Jurgens $\mathrm{H}$ and Winkler K: Prognostic factors in high-grade osteosarcoma of the extremities or trunk: An analysis of 1,702 patients treated on neoadjuvant cooperative osteosarcoma study group protocols. J Clin Oncol 20(3): 776-790, 2002. PMID: 11821461. DOI: $10.1200 /$ JCO.2002.20.3.776

5 Berner K, Hall KS, Monge OR, Weedon-Fekjaer H, Zaikova O and Bruland OS: Prognostic factors and treatment results of high-grade osteosarcoma in norway: A scope beyond the "classical" patient. Sarcoma 2015: 516843, 2015. PMID: 25784831. DOI: $10.1155 / 2015 / 516843$

6 Cates JM and Schoenecker JG: Proximal location in extremity long bones is a poor prognostic factor for osteosarcoma: A retrospective cohort study of 153 patients. Acta Oncol 55(8): 1036-1039, 2016. PMID: 27145235. DOI: 10.3109/0284186x.2016.1156740

7 Whelan JS, Jinks RC, McTiernan A, Sydes MR, Hook JM, Trani L, Uscinska B, Bramwell V, Lewis IJ, Nooij MA, van Glabbeke M, Grimer RJ, Hogendoorn PC, Taminiau AH and Gelderblom H: Survival from high-grade localised extremity osteosarcoma: Combined results and prognostic factors from three european osteosarcoma intergroup randomised controlled trials. Ann Oncol 23(6): 1607-1616, 2012b. PMID: 22015453. DOI: 10.1093/annonc/ mdr491

8 Jeon DG, Song WS, Cho WH, Kong CB and Cho SH: Proximal tumor location and fluid-fluid levels on mri predict resistance to chemotherapy in stage iib osteosarcoma. Clin Orthop Relat Res 472(6): 1911-1920, 2014. PMID: 24574120. DOI: 10.1007/s11999014-3521-1

9 Iwata S, Nakamura T, Gaston CL, Carter SR, Tillman RM, Abudu A, Jeys L and Grimer RJ: Diaphyseal osteosarcomas have distinct clinical features from metaphyseal osteosarcomas. Eur J Surg Oncol 40(9): 1095-1100, 2014. PMID: 25037733. DOI: 10.1016/j.ejso.2014.06.003

10 Berner K, Johannesen TB, Berner A, Haugland HK, Bjerkehagen $\mathrm{B}$, Bohler PJ and Bruland OS: Time-trends on incidence and survival in a nationwide and unselected cohort of patients with skeletal osteosarcoma. Acta Oncol 54(1): 25-33, 2015. PMID: 24957555. DOI: 10.3109/0284186x.2014.923934

11 Fletcher CDM, Bridge JA, Hogendoorn PCW and Mertens F: Who classification of tumours of soft tissue and bone, 4th ed. International Agency for Research on Cancer: Lyon, 2013.

12 Saeter G, Alvegard TA, Elomaa I, Stenwig AE, Holmstrom T and Solheim OP: Treatment of osteosarcoma of the extremities with the t-10 protocol, with emphasis on the effects of preoperative chemotherapy with single-agent high-dose methotrexate: A scandinavian sarcoma group study. J Clin Oncol 9(10): 1766-1775, 1991. PMID: 1717666. DOI: 10.1200/JCO.1991.9.10.1766

13 Enneking WF, Spanier SS and Goodman MA: A system for the surgical staging of musculoskeletal sarcoma. Clin Orthop Relat Res 153: 106-120, 1980. PMID: 7449206.

14 Cho WH, Song WS, Jeon DG, Kong CB, Kim MS, Lee JA, Yoo JY, Kim JD and Lee SY: Differential presentations, clinical courses, and survivals of osteosarcomas of the proximal humerus over other extremity locations. Ann Surg Oncol 17(3): 702-708, 2010. PMID: 19921336. DOI: 10.1245/s10434-0090825-6 
15 Bramer JA, van Linge JH, Grimer RJ and Scholten RJ: Prognostic factors in localized extremity osteosarcoma: A systematic review. Eur J Surg Oncol 35(10): 1030-1036, 2009. PMID: 19232880. DOI: 10.1016/j.ejso.2009.01.011

16 Meyers PA, Heller G, Healey J, Huvos A, Lane J, Marcove R, Applewhite A, Vlamis V and Rosen G: Chemotherapy for nonmetastatic osteogenic sarcoma: The memorial sloan-kettering experience. J Clin Oncol 10(1): 5-15, 1992. PMID: 1370176. DOI: doi.org/10.1200/JCO.1992.10.1.5

17 Rosen $G$ and Nirenberg A: Neoadjuvant chemotherapy for osteogenic sarcoma: A five year follow-up (t-10) and preliminary report of new studies (t-12). Prog Clin Biol Res 201: 39-51, 1985. PMID: 3867902.
18 Friebele JC, Peck J, Pan X, Abdel-Rasoul M and Mayerson JL: Osteosarcoma: A meta-analysis and review of the literature. Am J Orthop 44(12): 547-553, 2015. PMID: 26665241.

19 Luetke A, Meyers PA, Lewis I and Juergens H: Osteosarcoma treatment - where do we stand? A state of the art review. Cancer Treat Rev 40(4): 523-532, 2014. PMID: 24345772. DOI: 10.1016/j.ctrv.2013.11.006
Received March 1, 2019

Revised April 1, 2019

Accepted April 2, 2019 